\title{
Diet-Derived Post-Biotic Metabolites to Promote Microbiota Function and Human Health
}

\author{
Bharati Patel ${ }^{1}$, Kadamb Patel ${ }^{2}$ and Shabbir Moochhala*1,3 \\ ${ }^{1}$ Department of Surgery, Yong Loo Lin School of Medicine, National University of Singapore, Singapore \\ ${ }^{2}$ Centre for Molecular Diagnostics, Temasek Polytechnic, Singapore \\ ${ }^{3}$ Department of Pharmacology, Yong Loo Lin School of Medicine, National University of Singapore, Singapore \\ *Corresponding author: Shabbir Moochhala, Department of Surgery, Yong Loo Lin School of Medicine, Singapore
}

\section{ARTICLE INFO}

Received: 唪 June 10, 2020

Published: 慧 June 18, 2020

Citation: Bharati Patel, Kadamb Patel, Shabbir Moochhala. Diet-Derived Post-Biotic Metabolites to Promote Microbiota Function and Human Health. Biomed J Sci \& Tech Res 28(2)-2020. BJSTR. MS.ID.004637.

Keywords: Microbiota; Postbiotics; Probiotics; Prebiotics; Fecal Microbiota Transplant (FMT); Dysbiosis; NGS-Next Generation Sequencing

Abbreviations: FMT: Fecal Microbiota Transplant; SCFA: Short Chain Fatty Acids; GI Tract: Gastrointestinal Tract; IBS: Irritable Bowel Syndrome; IBD: Inflammatory Bowel Disease; NGS: Next Generation Sequencing

\section{ABSTRACT}

The microbiome is a complex ecosystem, and it is unique to every individual. Recently, microbiota is known as hidden organ in its own right. It is a challenge to explore the interaction of this organ with our body for its influential role in human health. Recently, research on microbiome science is progressing rapidly. With the advanced application of Next Generation Sequencing (NGS), the genetic composition of microbiome is available. NGS provides mechanistic insights about the type of beneficial microbial species and which species detriments to one's health. It is now feasible to provoke a fingerprint of the whole microbiota of any given environment. Microbiota dysbiosis in conditions like Crohn's disease, neurological disorders to cancer has reported through progressive scientific research. Therefore, it is becoming necessary to restore or strengthen the intestinal microbiota environment through diet-based approaches such as probiotics, prebiotics, or fecal microbiota transplant proposed for combating the onset or progression of these diseases.

Results from NGS studies are now integrated into the clinical research and considering each microbiome as unique for an individual representing how NGS has a substantial impact on the future into personalized medicines against various diseases. In this mini review, we acknowledge the beneficial concept of postbiotics for the manipulation of the Intestinal Microbiota; Postbiotics are functional bioactive compounds, generated in a matrix during fermentation, which may use to promote health. The identification of new postbiotics and the pathways responsible for their production should lead to an improved mechanistic understanding of specific probiotics, prebiotics, and postbiotics in restoring intestinal microbiota composition as well as function. We are speculating that different types of postbiotic metabolites have potential applications in human health.

\section{Mini Review}

The human gastrointestinal tract (GI-tract) is composed of a microbial habitat, representing the site with a dynamic and complex mutualistic relationship between the gut microbiota and the host. The gut microbiota enhances the multitude of physiological functions in host with this mutual communication; the host's immune system often mediates this role [1]. Individual representatives of the gut microbiota also yielding a wide range of components that can be utilized by the host as well as by some other

microbial species present. These mutual associations identified as the host-microbe and microbial community intercommunications [2]. There are several ways known to modulate the functional as well as compositional aspects of gut microbiota. The term '-biotics' means a strategy applied to nutrition favoring the gut microbiota for the host health in a most favorable state. The 'biotic' is a term assigned from the Greek word bi-otikós, mentions 'pertaining to life', which illustrates to the bioecological community composed of living organisms together with their physical environment [3]. 


\section{Postbiotics: A Relative Newcomer to Influence the} Function of the Microbiome and Human Health

The composition and functional aspects of gut microbiota have modulated by prebiotics, probiotics, and/or synbiotics. It also directly affects the immune response. In the members of the biotics family, a term called postbiotics is newly introduced. This term introduces to bioactive compounds, which produced during the fermentation process from the food-grade microorganisms. Postbiotics include metabolites, cell constituents, and microbial cells [4]. The metabolic byproducts (soluble factors), released upon bacterial lysis or secreted by live bacteria are referred to as postbiotics, such as Short Chain Fatty Acids (SCFA), enzymes, peptides, teichoic acids, peptidoglycan-derived muropeptides, polysaccharides, cell surface proteins, and organic acids [5,6]. As mentioned earlier, when considering a molecular mechanism, the interaction between host and microbial products seems to mediate the effect of postbiotics, triggers the immune system of the host, and thereby triggering, e.g., anti-inflammatory responses [7].

Several in vitro studies have performed to describe these molecular mechanisms; however, it is not fully understood the mechanism of action leading to these benefits in humans [8]. The challenging properties of postbiotics are long shelf life, safety dose parameters and its chemical structure. The content of different signaling molecules with anti-obesogenic, anti-proliferative, antiinflammatory, antioxidant and immunomodulatory activity have drawn a major attention in the microbiome research [9]. In short, considering these properties of postbiotics, it is possible that in the future, postbiotics may contribute to human health by improving the host's specific physiological functions. Further studies are required to understand this mechanism of action. Considering all the above criteria, this review aims to define the use of postbiotics and their possible benefits for overall human health.

\section{Advanced Strategies to Strengthen the Microbiota}

Usually, there is always a synergistic relationship between the gut microbiota and the host. However, when the ecosystem undergoes abnormal changes, gut bacteria turned into the stage, which is potentially harmful [10]. During dysbiosis between the gut bacteria in patients or animal models, it may cause many diseases. For example, treatment with antibiotics as well as surgery resulted in the pseudomembranous colitis because of the production of toxin by Clostridium difficile and the sepsis of Escherichia coli, Enterococcus faecalis and Enterococcus faecium, and intra-abdominal abscesses due to Bacteroides fragilis [11]. The microbial diversity is known as the presence of different bacterial strains in the gastrointestinal tract (GI tract). The main aspect of overall human health and has linked to various human diseases, like low bacterial diversity in obesity and inflammatory bowel disease [12,13]. To strengthen an emerging approach in microbiota, firstly, it is necessary to find out the molecules depleted into a particular disease condition, followed by supplementing the diet with either the depleted molecule or a precursor molecule which has converted into bioactive molecule via the community of microbes.

This is primarily a desirable approach because considering postbiotics are an essential class of functional units, and microbiota stimulates overall human health by utilizing them. The role of dietderived postbiotics in GI-tract and their possible mechanism of action concerning the various diseases on the microbiota metabolic profile has been investigated $[14,15]$. Microbiome research has demonstrated that the more the diversity of bacteria assimilating to our better health. The main critical factor determining your health is the diversity of microbiome, and it has demonstrated through numerous clinical trials that more microbial diversity indicates the better health $[16,17]$. It shows that a more diverse microbiome promotes good health because the different strains of probiotics produce various types of postbiotic metabolites. In different types of ecosystems like forests, grasslands, and the human microbiome, greater diversity equates the higher strength, resilience, and balance [18].

\section{Beginning of New Era in Microbiome Research-Postbiotic Metabolites}

Research on microbiome has gained in popularity recently because it is most probably considered as a vital element and play an essential role in human health with a bigger and auroral spotlight shining on it. More and more clinical studies are now focusing on a research investigation on the part of the microbiome for better visualization to prove the use of microbiome as therapeutics in various diseases [19].

\section{Probiotics vs Probiotics vs Synbiotics vs Postbiotics Probiotics}

Probiotics are live nonpathogenic microorganisms, and they are administered to enhance the microbial balance, particularly in the gastrointestinal tract, mainly consist of Saccharomyces boulardii yeast or lactic acid bacteria, like Lactobacillus and Bifidobacterium species. Probiotics are usually regulated as dietary supplements and foods and exert their profound beneficial effects through various mechanisms, including lowering intestinal $\mathrm{pH}$, decreasing colonization and invasion by pathogenic organisms, and tremendously modify the host immune response [20].

\section{Prebiotics}

Unlike probiotics, prebiotics are non-viable food ingredients, and they are metabolized selectively by the beneficial intestinal microbial species. The microbiota modulated by a diet supplemented with prebiotics is designed explicitly for our better health and immune by stimulating the numbers as well as improved functions of lactobacilli and bifidobacteria. The 'optimal' gut microflora is essential for strengthening the resistance against virulent or pathogenic bacteria; in turn, the immune response then stimulates the lower level of blood ammonia and reduced threatening of various diseases including cancer [21]. 


\section{Synbiotics}

It showed in various investigations when a microbial product contains the mixture of both probiotics and prebiotics, it is defined by the term symbiotic, which means synergy. An example The product containing oligofructose and probiotic bifidobacteria would fulfill the definition of synbiotics. It is still possible to argue that synergism is achieved in vivo by the ingestion of lactobacilli on the one hand and the promotion of indigenous bifidobacteria on the other hand [22].

\section{Postbiotics}

Postbiotics, defined as functional bioactive compounds generated in a matrix during fermentation, which may be used to promote health. The term postbiotics considered an umbrella term for all synonyms and related phrases of these microbial fermentation components. Other nutritional components, when applied along with postbiotics to accelerate the health, may act the way to increase the potency of active microorganisms or converting them into the functional ingredients. With this emerging concept of postbiotics and a combination of the microbiome may create food, homeostasis of microbes, and personalized treatment targeting various diseases bring even close together to work [23]. The takeaway for postbiotics is that while they are a new (and exciting) consideration for understanding microbiome homeostasis, our knowledge for their mechanism and action is mostly unexplored, and current utilization remains in a specialized clinical setting. Researchers believe that postbiotics may be a smart alternative to administer whole bacteria (in a probiotic form) for certain people suffering from inflammatory conditions. Postbiotics can diminish the inflammation; this may maintain colonic and intestinal homeostasis, postbiotics might be the next wave of supplements used to foster better gut health [4].

Mainly the postbiotics derived from Lactobacillus and Bifidobacterium strains have been used in the majority of cases. There are other bacterial species like Streptococcus and Faecalibacterium proved as a source of postbiotics [5,6]. The protective mechanism on the endothelial function has not been elucidated; however, this could be due to changes in the gut microbiota and its metabolic byproducts; the restoration of the gut barrier function, and the effects on endotoxemia, inflammation, and renal sympathetic nerve activity [24]. The microbiota produces SCFAs, considered as a class of bioactive and beneficial molecules. A study performed in humans subjects of African origin suffering from colon cancer with low and higher risk and focusing on to observe a possible correlation and comparisons between the metabolites and colonic microbes. The results demonstrated that there was a decrease in SCFA production, bile acid metabolites of bacterial origin, indicating the higher the risk of colon cancer [25]. The abundance of butyrate, acetate, and propionate has also changed. It is correlated with the health deterioration of elderly patients, further underscoring the importance of bacterial SCFA production in GI-tract physiology [26].

\section{Some of the Better Known Postbiotic Metabolites include the Following}

a) B-vitamin synthesis biotin, cobalamin, folates, nicotinic acid, pantothenic acid, pyridoxine, riboflavin, and thiamine [27]

b) Vitamin $\mathrm{K}[27]$

c) Short-chain fatty acids (SCFAs): The undigested carbohydrates produce acetate, butyrate, and propionate (SCFA) through fermentation process in GI-tract. It helps in nourishing with a significant source of energy to the colon, also helps in intestinal growth and differentiation. SCFA also showed an essential impact on many metabolic processes [28].

d) Glutathione: synthesized by Lactobacillus-fermentum ME3 [29]

e) Antimicrobial peptides (AMPs) [30]

f) Phenylacetic acid [31]

g) D-amino acids [32]

h) Hydrogen peroxide [33]

i) Volatile organic compounds (VOCs) [34]

j) Phytoestrogens: Equol, enterolactone, enterodiol [35]

k) Urolithin A and urolithin B [36]

I) Fulvic acids [37].

\section{How are Postbiotics Beneficial for Microbiota and Humans?}

Currently, increased understanding on health and role of the gut microbiota has highlighted the benefits of pre- and probiotics, for the development of a stable and sound immune system in human, use of postbiotics are now challenging. Postbiotics may have a significant role in the adaptive immune system as well as it may act to improve the hut barrier function [6,38], including short-chain fatty acids (SCFAs), polysaccharides, enzymes, peptides (proteins), cell surface proteins, and vitamins [39].

\section{Helps in the Growth of Probiotics - Beneficial Microbial Species}

Lactic acid bacteria produce postbiotics through metabolic processes in our body, simulating activities by simulating probiotic activities in specific ways. It demonstrated that the lactic acid bacteria, already existing into the microbiome supported by postbiotics have specific essential properties like they induce support in removing the heavy metals from our body and also reduces the effect of viruses and toxins [40]. They also mimic the potential therapeutics and beneficial effects of probiotics while 
avoiding the risk of administering live microorganisms to the patients who cannot accept them when having existing immature intestinal barriers or weaken immune defenses. Importantly, postbiotics have a unique identity in beneficial effects on the host. It accelerates intestinal barrier maturation and healing in a condition where the probiotic bacteria that are killed due to heat in the gastrointestinal tract may act as a postbiotics by retaining their structure [41] and supports the healing against weak immune defenses.

\section{Reduces the Presence of Harmful Pathogens}

Our body is home to both types of bacteria, e.g., beneficial as well as the harmful bacteria. It is well known that some natural substances like certain herbs and plants inheriting antimicrobial properties help in reducing the effect of pathogenic bacteria, and due to this antimicrobial property, they are useful in preventing the infections and thereby illnesses. Several research publications illustrated that postbiotics might have the same antimicrobial abilities, and that is the reason now to use postbiotics in the next frontier for immune response support against the harmful pathogens. Examples of the postbiotics that capable of reducing the effects of pathogens include listeria monocytogenes, clostridium perfringens, salmonella enterica, and Escherichia coli bacterial species.

\section{It lowers Inflammatory Diseases as well as Oxidative Stress}

lactobacillus casei DG (LC-DG), a probiotic bacteria functions together with microbiota produces a byproduct that is beneficial to our body. These byproducts support to modulate the inflammatory/ immune responses. [42], like inflammatory suppression, reduced generation of reactive oxygen species, and regulation of apoptosis. It also supports our body to recover faster against the symptoms of IBS and IBD.

\section{Conclusion}

Postbiotics comprise metabolites and cell-wall components, secreted by live bacteria or released after bacterial lysis, with beneficial activities in the host. Postbiotics may induce anti inflammatory, immunomodulatory, anti-obesogenic, as well as the antioxidant activities, and due to these properties, postbiotics may contribute to the improvement of host health by providing specific physiological effects, even though the exact mechanisms are not fully understood. Additional efforts are necessary to allow discovery and characterization of new postbiotics, which may contribute to the understanding of the signaling pathway modulation. Novel research will allow the generation of detailed information to ensure stability during the manufacturing processes of postbiotic products and their efficacy. Particular attention should require in the development of uniform and stringently defined culture procedures to eliminate possible variability of postbiotics production since uncontrolled environmental factors can well change metabolism and undergo unexpected transient variability. Beside, well-designed randomized placebo-controlled human/clinical intervention trials, along with metabolomics study, must be conducted looking to support health claims of postbiotics supplementation.

\section{References}

1. Honda K, Littman DR (2016) The Microbiota in adaptive immune homeostasis and disease. Nature 535(7610): 75-84.

2. Fischbach MA, Segre JA (2016) Signaling in host-associated microbial communities. Cell 164(6): 1288-1300.

3. Dictionary.com. Biotic.

4. Wegh CAM, Geerlings SY, Knol J, Roeselers G, Belzer C (2019) Postbiotics and Their Potential Applications in Early Life Nutrition and Beyond. Int J Mol Sci 20(19): 4673.

5. Konstantinov S, Kuipers E, Peppelenbosch MP (2013) Functional genomic analyses of the gut microbiota for CRC screening. Nat Rev Gastroenterol Hepatol 10(12): 741-745.

6. Tsilingiri K, Rescigno M (2013) Postbiotics: What else? Beneficial Microbes 4(1): 101-107.

7. Gosálbez L, Ramón D (2015) Probiotics in transition: novel strategies. Trends Biotechnol 33(4): 195-196

8. Caroline N de Almada, Carine N Almada, Rafael CR Martinez, Anderson S Sant'Ana (2016) Paraprobiotics: Evidences on their ability to modify biological responses, inactivation methods and perspectives on their application in foods. Trends in Food Science \& Technology 58: 96-114.

9. Aguilar Toalá JE, Garcia Varela R, Garcia HS, Mata Haro V, González Córdova AF, et al. (2018) Postbiotics: An evolving term within the functional foods field. Trends in Food Science \& Technology 75: 105-114.

10. Petersen C, Round JL (2014) Defining dysbiosis and its influence on host immunity and disease. Cell Microbiol 16 (7): 1024-1033.

11. Wilcox MH (2003) Clostridium difficile infection and pseudomembranous colitis. Best Pract. Res Clin Gastroenterol 17(3): 475-492.

12. Turnbaugh PJ, Hamady M, Yatsunenko T, Cantarel BL, Duncan A, et al. (2009) A core gut microbiome in obese and lean twins. Nature 457: 480484 .

13. Qin J, Li R, Raes J, Arumugam M, Burgdorf KS, et al. (2010) A human gut microbial gene catalog established by metagenomic sequencing. Nature 464(7285): 59-65.

14. Selin Bolca, Tom Van de Wiele, Sam Possemiers (2013) Gut metabotypes govern health effects of dietary polyphenols. Current Opinion in Biotechnology 24(2): 220-225.

15. Marcobal A, Kashyap PC, Nelson TA, Aronov PA, Donia MS, et al. (2013) A metabolomic view of how the human gut microbiota impacts the host metabolome using humanized and gnotobiotic mice. ISME J 7(10): 1933-1943.

16. Tuddenham S, Sears CY (2015) The Intestinal Microbiome and Health Curr Opin Infect Dis 28(5): 464-470.

17. Claesson MJ, Jeffery IB, Conde S, Power SE, O'Connor EM, et al. (2012) Gut microbiota composition correlates with diet and health in the elderly. Nature 488(7410): 178-184.

18. Ross Pelton (2019) Naturopathic Medicine; Probiotics. Journal of natural medicine.

19. Patel RM, Denning PW (2013) Therapeutic use of prebiotics, probiotics, and postbiotics to prevent necrotizing enterocolitis: what is the current evidence? Clinics in perinatology 40(1): 11-25.

20. Nancy Toedter Williams (2010) Probiotics, American Journal of HealthSystem Pharmacy 67(6): 449-458. 
21. Manning TS, Gibson GR (2004) Prebiotics, Best Practice \& Research Clinical Gastroenterology 18(2): 287-298.

22. Jürgen Schrezenmeir, Michael de Vrese (2001) Probiotics, prebiotics, and synbiotics-approaching a definition, The American Journal of Clinical Nutrition 73(Suppl 2): 361S-364S

23. Wegh CAM, Geerlings SY, Knol J, Roeselers G, et al. (2019) Postbiotics and Their Potential Applications in Early Life Nutrition and Beyond. Int J Mol Sci 20: 4673.

24. Robles Vera I, Toral M, Romero M, Jiménez R, Sánchez M, et al. (2017) Antihypertensive effects of probiotics. Current Hypertension Reports 19(4): 26.

25. Junhai Ou, Carbonero F, Zoetendal EG, DeLany JP, Wang M, et al. (2013) Diet, Microbiota, and microbial metabolites in colon cancer risk in rural Africans and African Americans. The American Journal of Clinical Nutrition 98(1): 111-120.

26. Claesson M, Jeffery I, Conde S, Power SE, O'Connor EM, et al. (2012) Gut microbiota composition correlates with diet and health in the elderly. Nature 488(7410): 178-184.

27. Hill MJ (1997) Intestinal flora and endogenous vitamin synthesis. Eur Cancer Prev 6 Suppl 1: S43-S45.

28. Morrison DJ, Preeston T (2016) Formation of short chain fatty acids by the gut microbiota and their impact on human metabolism. Gut Microbes $7(3): 189-200$.

29. Mikelsaar M, Zilmer M (2009) Lactobacillus fermentum ME-3: an antimicrobial and anti-oxidative probiotic. Microb Ecol Health Dis 21(1): $1-27$.

30. Dobson A, Cotter PD, Ross RP, Hill C (2012) Bacteriocin Production: a Probiotic Trait? Appl Environ Microbiol 78(1): 1-6.

31. Ohhira I, Kuwaki S, Morita H, Suzuki T, Tomita S, et al. (2004) Identification of 3-phenyl lactic acid as a possible antibacterial substance produced by Enterococcus faecalis TH10. Biocontrol Science 9(3): 77-81.

32. Cava F, Lam H, de Pedro MA, Waldor MK (2011) Emerging knowledge of regulatory roles in D-amino acids in bacteria. Cell Mil Life Sci 68(5): 817-831.

ISSN: 2574-1241

DOI: 10.26717/BJSTR.2020.28.004637

Shabbir Moochhala. Biomed J Sci \& Tech Res

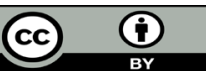

This work is licensed under Creative Commons Attribution 4.0 License

Submission Link: https://biomedres.us/submit-manuscript.php
33. Hertzberger R, Arents J, Dekker HL, Pridmore RD, Gysler C, et al. (2014) H2O2 Production in Species of the Lactobacillus acidophilus Group: a Central Role for a Novel NADH-Dependent Flavin Reductase. Appl Environ Microbiol 80(7): 2229-2239.

34. Bos LD, Sterk PJ, Schultz MJ (2013) Volatile Metabolites of Pathogens: A Systemic Review. PLoS Pathog 9(5): e1003311.

35. Frandenfeld CL, Atkinson C, Wähälä K, Lampe JW (2014) Obesity prevalence in relation to gut microbial environments capable of producing equol or $\mathrm{O}$-desmethylangolensin from the isoflavone daidzein. Eur J Clin Nutr 68(4): 526-530.

36. Larrosa M, González Sarrías A, García Conesa MT, Tomás Barberán FA Espín JC (2006) Urolithins, Ellagic Acid-Derived Metabolites Produced by Human Colonic Microflora, Exhibit Estrogenic and Antiestrogenic Activities. J Agric Food Chem 54(5): 1611-1620.

37. Fulvic acids: Biobank

38. Martin R, Nauta AJ, Amor KB, Knippels LMJ, Knol J, et al. (2010) Early life: gut microbiota and immune development in infancy. Benef Microbe 1(4): 367-382.

39. Aguilar Toalá JE, Garcia Varela R, Garcia HS, Mata Haro V, González Córdova AF, et al. (2018) Postbiotics: An evolving term within the functional foods field. Trends in Food Science \& Technology 75: 105-114.

40. Salminen S, Nybom S, Meriluoto J, Collado MC, Vesterlund S, et al. (2010) Interaction of probiotics and pathogens--benefits to human health? Curr Opin Biotechnol 21(2): 157-167.

41. Patel RM, Myers LS, Kurundkar AR, Maheshwari A, Nusrat A, et al. (2012) Probiotic bacteria induce maturation of intestinal claudin 3 expression and barrier function. Am J Pathol 180(2): 626-635.

42. Compare D, Rocco A, Coccoli P, Angrisani D, Sgamato C, et al. (2017) Lactobacillus casei DG and its postbiotic reduce the inflammatory mucosal response: an ex-vivo organ culture model of post-infectious irritable bowel syndrome. BMC Gastroenterol 17(1): 53.

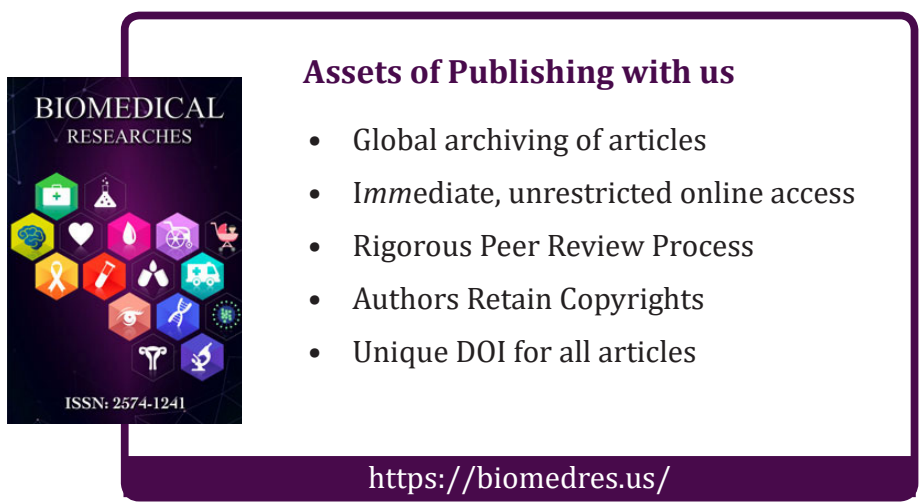

